\title{
THE BIOACCUMULATION AND METABOLIC EFFECTS OF CIPROFLOXACIN-HCL AND CIPROFLOXACIN FREE BASE IN YELLOW LUPIN (Lupinus luteus L.) SEEDLINGS
}

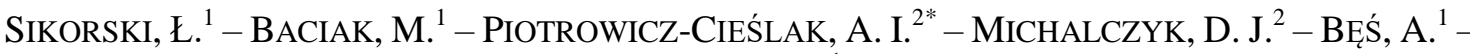 \\ ADOMAS, B. ${ }^{1}$ \\ ${ }^{I}$ Department of Environmental Toxicology, Faculty of Environmental Management and \\ Agriculture, University of Warmia and Mazury in Olsztyn \\ Prawocheńskiego 17, 10-720 Olsztyn, Poland \\ ${ }^{2}$ Department of Plant Physiology, Genetics and Biotechnology, Faculty of Biology and \\ Biotechnology, University of Warmia and Mazury in Olsztyn \\ Oczapowskiego 1A, 10-718 Olsztyn, Poland \\ *Corresponding author: \\ e-mail: acieslak@uwm.edu.pl
}

(Received $4^{\text {th }}$ May 2017; accepted $2^{\text {nd }}$ Aug 2017)

\begin{abstract}
Two forms of ciprofloxacins (CIP) are used in animal husbandry and veterinary medicine, and may occur in manure-fertilized soil as pollutants: $\mathrm{CIP}-\mathrm{HCl}$ salt, readily dissolvable in water, and the free base CIP, practically water-insoluble. The aim of this study was to compare the phytotoxicity of both forms of CIP to yellow lupin seedlings (Lupinus luteus L.). In eight-day-old lupin seedlings higher amounts of both water-soluble and insoluble CIP were detected in roots, compared to shoots. The amount of CIP in plants treated with CIP-HCl was 1.5 times higher compared to plants treated with the insoluble, free base form. Nevertheless, both forms of the antibiotic caused very similar reduction in lupin shoot length, root length and seedling fresh mass as well as very similar increases in seedling dry mass. Activity of guaiacol peroxidase in control seedlings was 14.09 and $49.25 \mathrm{U}$ in roots and shoots, respectively, and in plants treated with CIPs it was highly dependent on the antibiotic dose. Guaiacol peroxidase activity was stimulated in plants treated with $5 \mathrm{mM} \mathrm{CIP}$ of any kind (water-soluble or insoluble), and this activation was particularly visible in roots, while the enzyme activity decreased to nearly zero in both roots and shoots of plants treated with CIPs at the level of or above $20 \mathrm{mM}$. The activity of superoxide dismutase in roots was severely repressed by even the lowest dose of any CIP, while it was slightly stimulated in shoots by any of the CIPs at the level not exceeding $10 \mathrm{mM}$. The following carbohydrates were detected in roots of lupin grown in soil contaminated with CIPs (water-soluble and insoluble): Dchiro-inositol, D-pinitol, myo-inositol, galactose, glucose and sucrose. The content of myo-inositol and sucrose in lupin roots increased with increasing concentrations of soluble and insoluble CIPs. Neither of the forms of the drug clearly affected the content of D-chiro-inositol, D-pinitol, glucose and galactose. Both forms of CIP showed very similar effects on lupin seedlings, however, the accumulation of CIP$\mathrm{HCl}$ was a little higher compared to the free base form of this antibiotic.
\end{abstract}

Keywords: lupin, plant uptake, guaiacol peroxidase and superoxide dismutase activity, soluble carbohydrates

\section{Introduction}

Antibiotics are widely applied in human and veterinary medicine and industrial animal production. The increased usage of well-known forms of these pharmaceuticals, as well as the launch of new antibiotics and the spread of multi-stand industrial farms have resulted in increased environmental pollution with antibiotics and their metabolites. The environmental impact of the majority of these drugs is unknown or is regarded as low. However, their presence may be linked to ecotoxicological changes with negative effects on ecosystems (Ding and He, 2010; Santos et al., 2010). 
Ciprofloxacin (CIP) belongs to the fluoroquinolone group of pharmaceuticals and is one of the most commonly applied antibiotics. It has a broad spectrum of biological activity and is used against both Gram-negative and Gram-positive bacteria. When applied frequently, it infiltrates soil and is detected at the level 6 to $750 \mu \mathrm{g} / \mathrm{kg}$ (Golet et al., 2002; Kemper, 2008; Martínez-Carballo et al., 2007). The mechanism of CIP toxicity towards bacteria involves the inhibition of DNA topoisomerases: topoisomerase II (DNA gyrase) and topoisomerase IV, resulting in an arrest of bacterial DNA replication (Oliphant and Green, 2002). The bioavailability of ciprofloxacin in various mammals is estimated at about $70 \%$. After oral ingestion, about $15 \%$ of the drug undergoes transformation into less active metabolites that are detected in urine, while $40-50 \%$ is detected as the unchanged form (DrugBank Reports, DB00537).

Drug bioavailability is affected by several factors. These include the properties of the drug itself, as well as the type and quantity of the solvent. Many antibiotics may occur as water soluble salts - hydrochlorides - and water-insoluble, free base forms (Caco et al., 2008). The kinetics of the drug dissolution process affects the drug absorption and accumulation in plants. Plants absorb antibiotics from water and soil and distribute them throughout plant tissues as a result of passive transport in transpiration stream (Liu et al., 2013). Antibiotics in plants may decrease the plant growth rate and activity of biochemical defence reactions (Sikorski at al., 2014). One of the key defence mechanisms in plants is activation of antioxidative enzymes: guaiacol peroxidase (POD, EC 1.11.1.7) and superoxide dismutase (SOD, EC 1.15.1.1). These enzymes are present in nearly all plants and they are important elements of plant antioxidative protection system (Cheng et al., 2012; Passardi et al., 2004; Cesarino et al., 2013). They catalyse the disproportionation of the superoxide $\mathrm{O}_{2}^{-}$radical into hydrogen peroxide and molecular oxygen. The reaction occurs in all organisms that metabolise oxygen. Class III peroxidases are secreted glycoproteins that participate in lignin and suberin biosyntheses and deactivate toxic compounds. Moreover, they are involved in the developmental processes by controlling auxin level and cell elongation (Passardi et al., 2004; Bakalovic et al., 2006).

Plant responses to some environmental stresses include the accumulation of soluble carbohydrates, that ensure the right cellular osmotic potential and create a protective layer around macromolecules. They also act as signal substances and take part in antioxidative defence (Noiraud et al., 2001; Rontein et al., 2002; Piotrowicz-Cieślak and Adomas, 2012).

The aim of the study was to compare phytotoxicity of two forms of CIP (watersoluble and insoluble) towards lupin (Lupinus luteus L.), cultivar Mister, by assessing selected morphological and biochemical features: seedling growth, the activity of guaiacol peroxidase, superoxide dismutase and the content of soluble carbohydrates as well as quantifying content of both drug forms in lupin roots and shoots.

\section{Material and Methods}

\section{Plant material and growth conditions}

Seeds of yellow lupin (Lupinus luteus L.) cv. Mister were germinated for eight days in Phytotoxkit plates (MicroBio Test, Inc., Belgium). Germination was carried out under controlled climatic conditions with temperature set at $25^{\circ} \mathrm{C}$ and $90 \%$ relative humidity (RH), with a 16h/8h day/night photoperiod and $3.4 \mathrm{klx}$ light intensity. Ninety milliliters of soil (sand, vermiculite, peat 1:0.3:1, v/ v/v) were placed in each plastic 
microbiotest plate. The soil was covered with Whatman No. 1 filter paper and watered with $27 \mathrm{ml}$ distilled water supplemented with water-soluble and insoluble CIP (SigmaAldrich) at final concentrations of $5 ; 10 ; 20 ; 40,80 \mathrm{mM}$. The control plants were watered with pure distilled water. The root length was estimated after eight days of germination using Image Tool for Windows. Fresh weight of seedlings was determined according to standard seed testing recommendations (ISTA, 1999). The experiment was carried out in four replicates, each containing 40 seedlings.

\section{Water-soluble and insoluble ciprofloxacin content in seedlings}

CIP was extracted from eight days-old seedlings grown in soil containing CIP-HCl or CIP free base. Plants were first rinsed with distilled water, then manual solid phase extraction (SPE) was carried out by squeezing the sap from plant material with a mortar and pestle and transferring the sap to SPE cartridges (Chromabond $®$ Easy, $3 \mathrm{ml} \times 200$ $\mathrm{mg}^{-1}$, Macherey-Nagel, Dtiren, Germany) prewashed with methanol (Rydzyński et al., 2017). After loading the samples to cartridges the antibiotic was eluted with $250 \mu 1$ methanol. CIP in seedlings was analyzed by HPLC according to Pailler et al. (2009) with small modifications. Briefly, the chromatographic system consisted of a Water Aliance 2695 HPLC system (Waters Corp.) with a binary high-pressure gradient pump, an automatic injector and a column oven. The chromatographic column was an Atlantis T3 column $(150 \times 3.0 \mathrm{~mm}, 3 \mu \mathrm{m})$ (Waters Corp.) at $40^{\circ} \mathrm{C}$. The MS-MS analyser consisted of Quattro micro ${ }^{\circledR}$ API MS (Waters Corp.) using electrospray in the positive mode (ESI+). $\mathrm{N}_{2}$ was used as nebulizer, drying, curtain and collision gas. A chromatographic gradient was applied for the separation of the analytes depending on the ionization mode employed, with a total chromatographic run of $18 \mathrm{~min}$. Gradient elution was carried out with aqueous $0.1 \%$ formic acid : $0.1 \%$ formic acid in acetonitrile at a flow rate of $4.5 \mathrm{ml} \times \mathrm{min}^{-1}$. Validation of the method included the assessment of selectivity, linearity $\left(1\right.$ to $\left.11 \mu \mathrm{g} \times \mathrm{ml}^{-1}\right)$, limits of detection $\left(8 \mathrm{ng} \times \mathrm{ml}^{-1}\right)$ and quantification

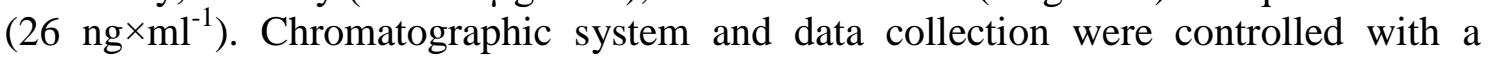
MassLynx 4.1. chromatographic software interfaced to a personal computer.

\section{Guaiacol peroxidase activity}

Extracts used to determine guaiacol peroxidase (POD) activity were prepared on ice. The root and shoot tissues $(500 \mathrm{mg}$ ) that grew in soil with water or with chlortetracycline were homogenized for 30 minutes in the extraction buffer $(0.1 \mathrm{M}$ Tris/HCl, pH 7.8, 8.75\% polyvinyl pyrrolidone, $0.1 \mathrm{M} \mathrm{KCl}, 0.28 \%$ Triton $\mathrm{X}-100$ ). Samples were centrifuged for 30 minutes at $4000 \times \mathrm{g}$ at $4^{\circ} \mathrm{C}$. The supernatant was passed through membrane filters with $0.45 \mu \mathrm{m}$ porosity. The protein content in samples was determined with the Lowry et al. (1951) protein assay. POD was determined based on the spectrophotometric detection (Cecil, CE2021 2000 Series) in a mixture containing $100 \mu \mathrm{l} 1 \%$ guaiacol, $2 \mathrm{ml} 0.1 \mathrm{M} \mathrm{KH}_{2} \mathrm{PO}_{4}, 150 \mu \mathrm{l}$ supernatant and $20 \mu \mathrm{l} 0.18 \% \mathrm{H}_{2} \mathrm{O}_{2}$. The absorption rate increase was measured at room temperature at the wavelength of $470 \mathrm{~nm}$. One unit of activity equals oxidation of $1 \mu$ mole $\mathrm{H}_{2} \mathrm{O}_{2} / \mathrm{mg}$ protein during 1 minute. POD analysis was carried out in four replications. 


\section{Superoxide dismutase activity}

Extracts used for superoxide dismutase (SOD) activity determination were prepared on ice. The root and shoot tissues $(200 \mathrm{mg}$ ) were homogenized in the extraction buffer $(1 \mathrm{ml})$ consisting of $50 \mathrm{mM}$ phosphate buffer ( $\mathrm{pH} 7.0$ ), $1 \%$ polyvinyl pyrrolidone, $0.1 \mathrm{M}$ $\mathrm{KCl}, 0.1 \%$ Triton $\mathrm{X}-100$. Samples were centrifuged for 15 minutes at $15000 \times \mathrm{g}$ at $4^{\circ} \mathrm{C}$, and supernatants were used as crude extracts for soluble protein quantification according to Lowry et al. (1951) with bovine serum albumin as the standard. Total SOD activity was assayed by the inhibition of the photochemical reduction of nitroblue tetrazolium (NBT). The reaction mixture $(2.2 \mathrm{ml})$ contained, $0.05 \mathrm{M}$ buffer $\mathrm{Na}_{2} \mathrm{CO}_{3} / \mathrm{NaHCO}_{3}$ (pH 10.2), 0.0001 M EDTA, $0.0001 \mathrm{M}$ xantin, $2.5 \times 10^{-5} \mathrm{M}$ NBT and $50 \mu 1$ of plant extract. The reduction of NBT by superoxide radicals to blue coloured formazan was followed at $560 \mathrm{~nm}$. One Unit of SOD activity is defined as that amount of enzyme required to inhibit the reduction of NBT by $50 \%$ under the specified conditions per $1 \mathrm{~g}$ protein.

\section{Soluble carbohydrates contents}

Soluble carbohydrates (sucrose, glucose, galactose, myo-inositol and D-chiroinositol) content in the seedlings were analyzed by GC chromatography according to Piotrowicz-Cieślak (2005). Tissues (100 mg fresh mass) were homogenized in ethanol : water mix, 1:1 (v/v) containing $300 \mu \mathrm{g}$ phenyl- $\alpha$-D-glucose as internal standard. The homogenate and the wash were combined in a $1.5 \mathrm{ml}$ microfuge tube, heated to $75^{\circ} \mathrm{C}$ for $30 \mathrm{~min}$ in order to inactivate endogenous enzymes and centrifuged at $15000 \times \mathrm{g}$ for 20 min. The supernatant was passed through a 10000 MW cut-off filter (Lida, Kenosha, WI USA). Aliquots of $0.3 \mathrm{ml}$ filtrate were transferred to silylation vials and evaporated to dryness under a stream of nitrogen. Dry residues were derived from $300 \mu \mathrm{l}$ of silylation mixture (trimethylsilylimidazole : pyridine, 1:1, v/v) in silylation vials (Thermo Scientific) at $70^{\circ} \mathrm{C}$ for $30 \mathrm{~min}$, and then cooled at room temperature. One $\mu \mathrm{l}$ soluble carbohydrate extract was injected into a split-mode injector of a Thermo Scientific gas chromatograph equipped with flame ionisation detector. Soluble carbohydrates were analyzed on a DB-1 capillary column (15 m length, $0.25 \mathrm{~mm}$ ID, $0.25 \mu \mathrm{m}$ film thickness, J\&W Scientific) and identified with internal standards as available. Concentrations were calculated from the ratios of peak area, for each analyzed soluble carbohydrates, to the peak area of respective internal standard. Quantities of soluble carbohydrates were expressed as mean \pm SD for four replications of each treatment.

\section{Statistical analysis}

The experiment was conducted in four replicates. The results were statistically evaluated using Statistica 6.0 software by performing analysis of variance (F test) for two factor experiments (split-plot) at the significance level $p=0.01$. The mean values of the plots were compared using q SNK test (Student-Newman-Keuls).

\section{Result}

The lupin root length was found to decrease due to the presence of the lowest of the tested CIP concentrations, i.e. $5 \mathrm{mM}$ for both drug forms (Figs 1A, 1B). CIP at the highest concentration $(80 \mathrm{mM})$, both as the soluble and insoluble form, significantly 
inhibited the growth of roots, by $85 \%$ and $75 \%$, respectively (Figs 1A, 1B). A similar reaction to the drug was observed for lupin shoots. Shoots of lupin growing in soil contaminated with $5 \mathrm{mM}$ of the water-soluble form of the drug were shorter by $7 \mathrm{~mm}$ on average than the shoots of plants growing in soil with the insoluble CIP form (Figs $1 A, 1 B)$. Fresh mass of roots growing in soil contaminated with $5 \mathrm{mM}$ of water-soluble and insoluble CIP was lower by $35 \mathrm{mg}$, compared with the control sample. On the other hand, the weight of fresh roots of plants growing in soil contaminated with soluble and insoluble CIP at the highest of the concentrations was lower by $116 \mathrm{mg}$ and $97 \mathrm{mg}$, respectively (Figs 1C, 1D). Fresh mass of lupin shoots also decreased, regardless of the drug form.

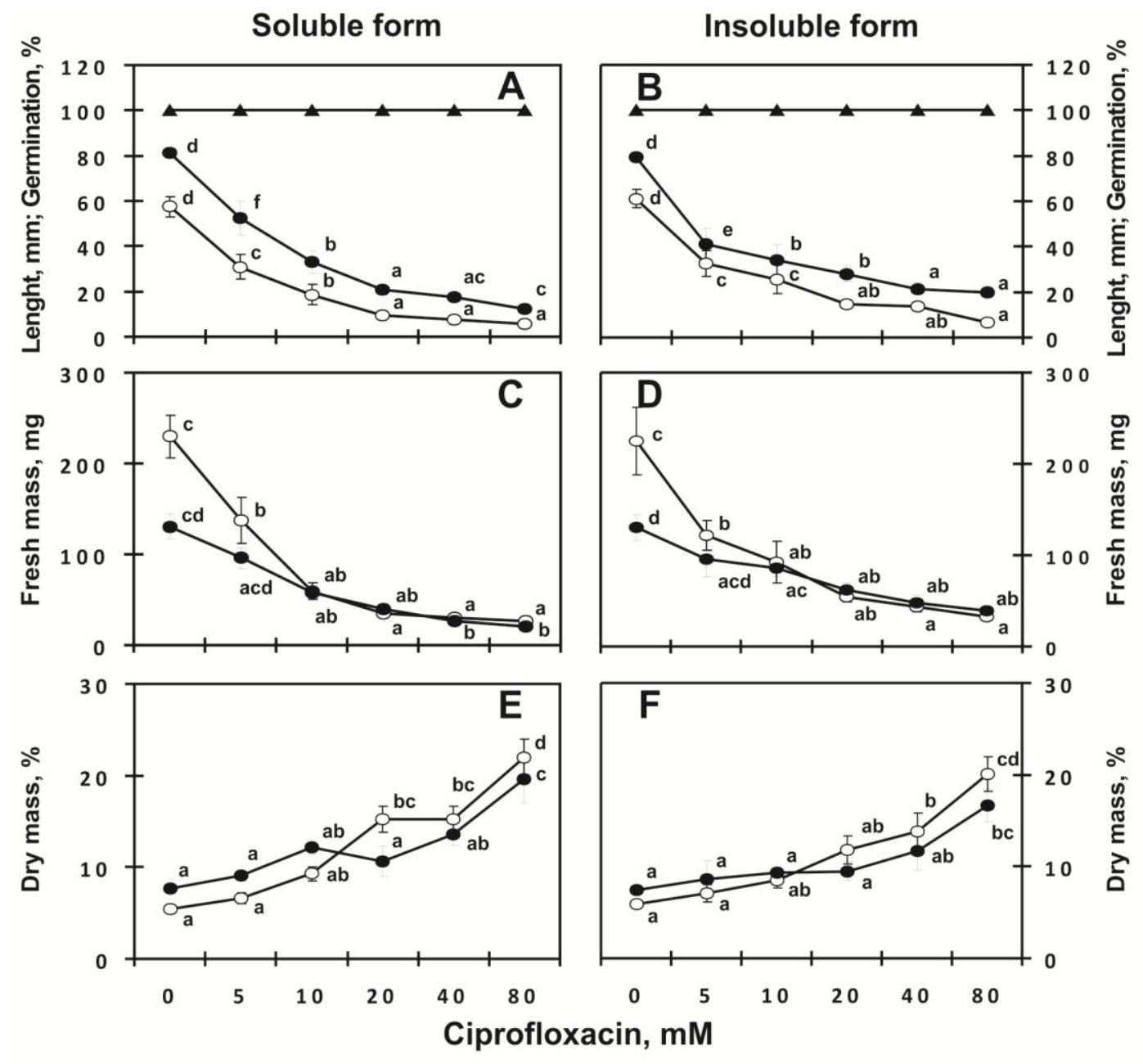

Figure 1. Panel $A$ and $B$ seedlings length ( $O$ - root, $\bullet-$ shoot), germination ( $\mathbf{\Delta})$; panel $C$ and $D$ fresh mass ( $($ - root, $\bullet$ - shoot); panel $E$ and $F$ dry mass $(O-$ root, $\bullet-$ shoot) in yellow lupin after three days on soil supplemented with different soluble and insoluble form of ciprofloxacin concentration $(0,5,10,20,40,80 \mathrm{mM})$. Data points represent the means $\pm S D$. Means with the same letter are not significantly different from each other $(p \leq 0.01)$.

Control shoots reached a fresh mass of $230 \mathrm{mg}$, whereas those from plants growing in soil contaminated with the drug water-soluble and insoluble forms at the highest concentrations reached only 27 and $32 \mathrm{mg}$ (Figs 1C, 1D). The dry mass of lupin roots 
increased with increasing concentrations of both forms of CIP in the soil. The dry mass of roots in the control sample was $7.6 \%$, whereas it was $19.6 \%$ and $16.6 \%$, (Figs $1 E$, $1 F)$, in the case of roots of plants growing in soil contaminated with $80 \mu \mathrm{M}$ of watersoluble and insoluble CIP, respectively. Similarly, the dry mass of shoots increased steadily with increasing contamination of soil with both forms of CIP. With the highest of the concentrations under study, it reached 22\% (water-soluble CIP) and 20\% (insoluble CIP) (Figs $1 E, 1 F)$.

CIP was found to be present in roots and shoots of seedlings treated with both CIP$\mathrm{HCl}$ and CIP free base (Fig. 2). The roots, in comparison to shoots, accumulated more CIP in both plants treated with the water-soluble and insoluble CIP form. The contents of the drug in roots of plants grown in soil with $5 \mu \mathrm{M}$ CIP (both CIP-HCl and CIP free base) were similar and averaged $200 \mu{\mathrm{g} \times \mathrm{g}^{-1}}$ fresh mass. Roots grown in soil contaminated with $80 \mu \mathrm{M}$ drug contained the highest amount of water-soluble CIP (700 $\mu \mathrm{g} \times \mathrm{g}^{-1}$ fresh mass) and insoluble CIP (400 $\mu \mathrm{g} \times \mathrm{g}^{-1}$ fresh mass). Compared to shoots, roots contained 1.5 less of both drug forms. Similar to root tissues, the contents of both drug forms in shoots depended on the level of CIP in soil. The maximum level of CIP in

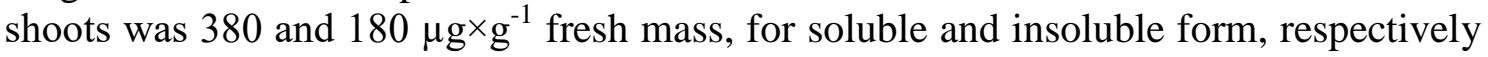
(Fig. 2). Lupin roots took up more of the drug than the shoots. The uptake of insoluble CIP from soil by yellow lupin roots and shoots was slower compared to the soluble form. The drug concentration in the lupin organs increased with increasing contamination of the soil with ciprofloxacin (Fig. 2).

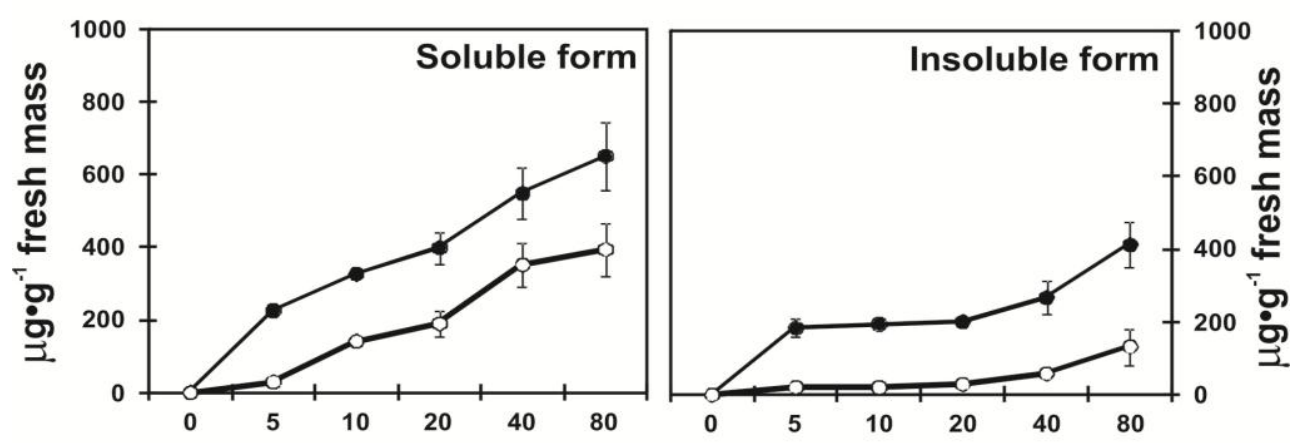

Ciprofloxacin, mM

Figure 2. Ciprofloxacin content in yellow lupin seedlings ( 0 - root, $\bullet-$ shoot) growing eight days on soil supplemented with different concentrations $(0,5,10,20,40,80 \mathrm{mM})$ of CIP-HCl or free $C I P$ base. Data points represent the means $\pm S D$ for three replicate samples.

The roots and shoots of lupin growing in control soil contained 14.09 and $49.25 \mathrm{U}$ of POD and 31.23 and $127.68 \mathrm{U}$ of SOD, respectively (Figs 3A, 3C). The activity of peroxidase increased to 17.59 and $19.23 \mathrm{U}$ in the roots of lupin growing in soil contaminated with the lowest of the concentrations of water-soluble and insoluble CIP (Figs 3A, 3B). The activity of the enzyme in roots was $78 \%$ lower than in the shoots of lupin exposed to water-soluble CIP $(5-10 \mathrm{mM})$, and $74 \%$ lower after exposure to the insoluble form (Figs 3A, 3B). The activity of peroxidase in lupin shoots, regardless of the drug concentration, was similar. Its highest level was found in lupin shoots growing in soil contaminated with $5 \mathrm{mM}$ CIP. An increase in POD activity of 38.22 and $31.55 \mathrm{U}$ was recorded in samples growing in soil contaminated with the soluble and insoluble 
form of the drug, respectively. Both forms CIP at $20 \mathrm{mM}$ concentration deactivated peroxidase in both the roots and shoots of lupin. The same tendency was observed for higher drug concentrations, i.e. 40 and $80 \mathrm{mM}$ (Figs 3A, 3B). Unlike peroxidase, the activity of SOD in lupin shoots was inhibited by water-soluble and insoluble CIP even at the lowest, i.e. $5 \mathrm{mM}$ concentration. The lowest concentrations of both CIP forms decreased the activity in shoots by 14.28 and $55.73 \mathrm{U}$, respectively. Shoots of plants growing in soil containing $80 \mathrm{mM}$ of soluble CIP showed a higher SOD activity by 17 U compared with shoots growing in soil with insoluble CIP. The SOD activity in shoots of plants growing in soil containing the highest concentration of soluble or insoluble CIP (80 mM) was lower by $58 \%$ and $72 \%$, compared with the control shoots (Figs 3C, $3 D$ ). On the other hand, in the roots, both forms of the drug at $10 \mathrm{mM}$ concentration had a slightly stimulating effect on SOD activity. The highest SOD activity was detected in roots growing in soil containing $10 \mathrm{mM}$ of soluble and insoluble CIP and it was 58.22 and $63.53 \mathrm{U}$, respectively.

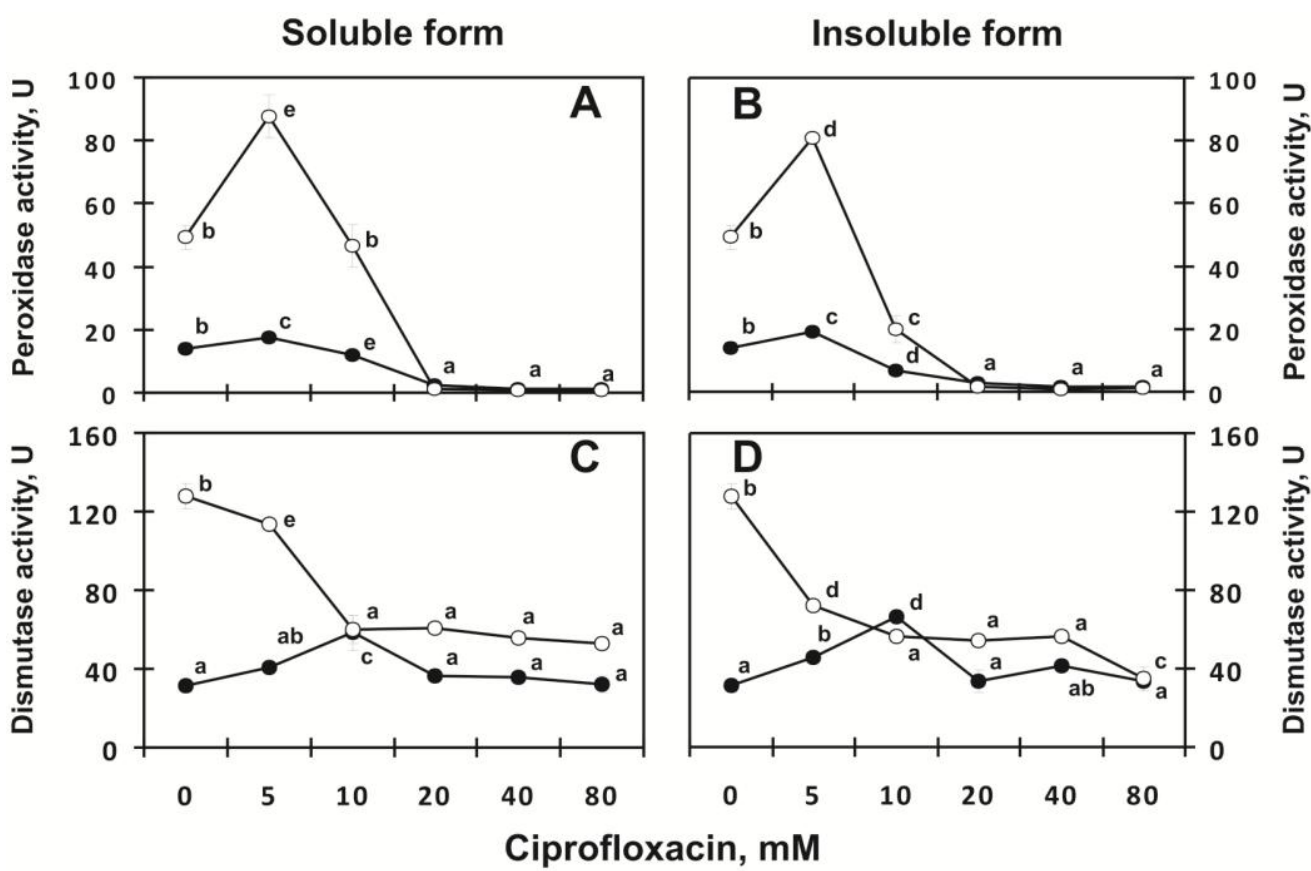

Figure 3. Panel $A$ and $B$ peroxidase and panel $C$ and $D$ dismutase activity in yellow lupin seedlings ( $\mathrm{O}$ - root, $\bullet-$ shoot) growing eight days on soil supplemented with different soluble and insoluble form of ciprofloxacin concentration $(0,5,10,20,40,80 \mathrm{mM})$. Data points represent the means $\pm S D$ for three replicate samples. Means with the same letter are not significantly different from each other $(p \leq 0.01)$.

Eight-day-old roots and shoots of lupin seedlings growing in soil contaminated with soluble or insoluble CIP were found to contain: D-chiro-inositol, D-pinitol, myoinositol, galactose, glucose, and sucrose (Figs $4 A-F)$. The content of D-chiro-inositol, D-pinitol, myo-inositol in control roots increased from $0.08,0.07$ and $0.34{\mathrm{mg} \times \mathrm{g}^{-1}}$ fresh

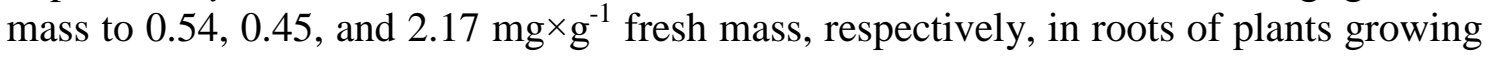
in soil contaminated with $80 \mathrm{mM}$ of soluble CIP (Fig. 4A).

The content of myo-inositol in roots growing in soil containing the highest concentration of insoluble CIP increased nearly four times. The roots contained an 
average of 10 times more glucose than galactose (Fig. 4C). The content of D-chiroinositol and D-pinitol in lupin shoots was not modified by either form of CIP. On the other hand, content of myo-inositol increased two-fold with the highest of the CIP concentrations under study (Figs 4A, 4B). No galactose was found in the tissues of shoots growing in soil with insoluble CIP at concentrations from 5 to $20 \mathrm{mM}$, whereas the concentration of glucose increased, and that of sucrose remained unchanged (Figs $4 D, 4 F)$. Neither the soluble nor the insoluble form of the drug modified the content of D-chiro-inositol or D-pinitol. Both of the CIP forms at the highest concentration increased the content of myo-inositol by two-fold (Figs 4A, 4B).

Soluble form
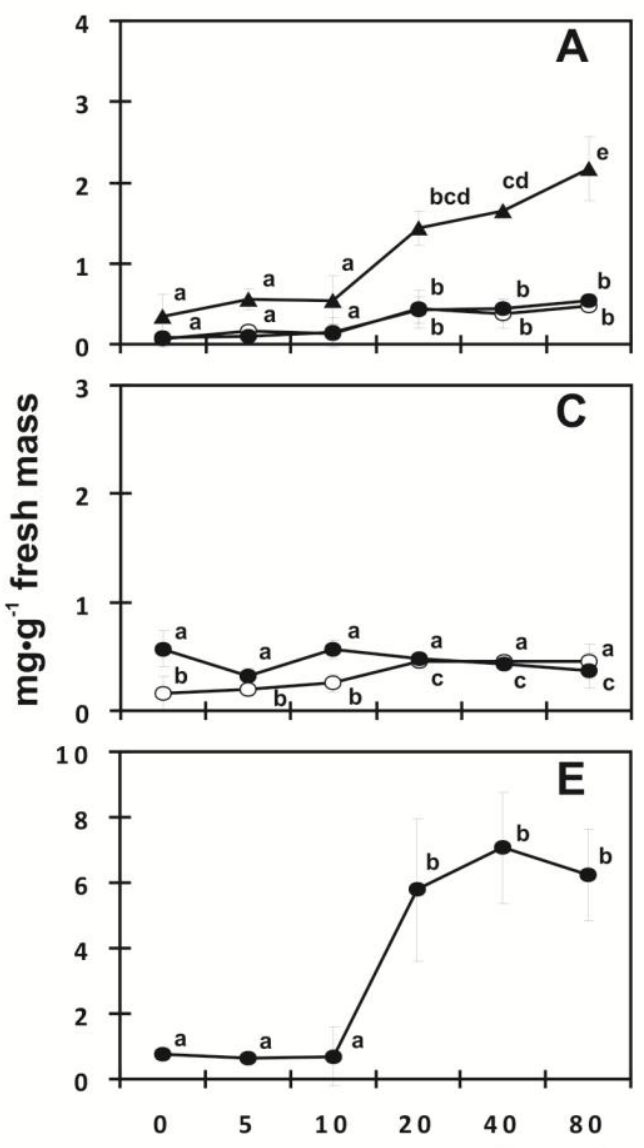

Insoluble form
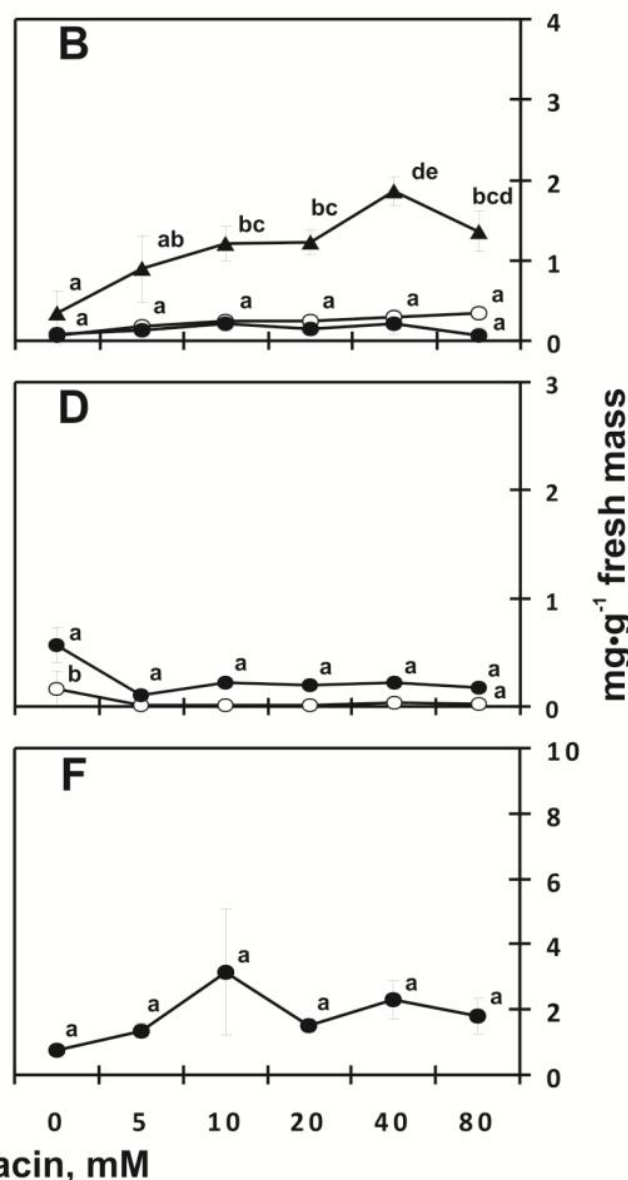

Figure 4. Panel A and B D-chiro-inositol - o, D-pinitol- •, myo-inositol- $\mathbf{\Delta}$ content; panel C and D galactose - $\bullet$, glucose - $O$ content; and panel $E$ and $F$ sucrose- $\bullet$ content in roots of yellow lupin growing eight days on soil supplemented with different soluble and insoluble form of ciprofloxacin concentration $(0,5,10,20,40,80 \mathrm{mM})$. Data points represent the means $\pm S D$ for three replicate samples. Means with the same letter are not significantly different from each other $(p \leq 0.01)$.

\section{Discussion}

Fluoroquinolones are absorbed by organic matter in the soil and in manure, as a result of which they degrade slowly and thereby tend to persist in the environment (Kemper, 2008; Lillenberg et al., 2010). When present in the environment, they delay 
water supply to seeds and inhibit germination (Jin et al., 2009). In the present study, soluble and insoluble CIP at concentrations from 5 to $80 \mathrm{mM}$, did not inhibit lupin seed germination (Figs 1A, 1B). According to Jin et al. (2009), Adomas et al. (2013), Ziółkowska et al. (2014) and Piotrowicz-Cieślak et al. (2010) seed germination is not a good indicator of the presence of drugs in the environment. Elongation of roots can be a better measure of soil contamination with drugs. Low concentrations of drugs in soil have a positive effect on plants and can even stimulate plant growth (and hormesis occurs at low concentrations) (Migliore et al., 2003). Only 100-fold increase of enroflaxacin concentration (from 50 to $5000 \mu \mathrm{g} \times \mathrm{L}^{-1}$ ) significantly decreases length of roots of plants such as cucumber (by $78 \%$ ), lettuce (83\%), beans $(83 \%)$, and radish (66\%) (Migliore et al., 2003). At the highest of the tested diclofenac concentrations (12 $\mathrm{mM}$ ), the rate of root growth retardation compared with roots of control seedlings amounts to 85,83 , and $80 \%$ for pea, lupin, and lentil, respectively (Ziółkowska et al., 2014). Doxycycline at $25 \mu \mathrm{g} / \mathrm{ml}$ concentration inhibited root elongation of Arabidopsis thaliana seedlings by $67 \%$ (Moullan et al., 2015).

The presence of soluble or insoluble CIP in soil reduced the length of lupin roots and shoots (Figs 1A, 1B). Shoots are shortened on average by 91, 86, and $77 \%$ for pea, lupin, and lentil, respectively, exposed to $12 \mathrm{mM}$ diclofenac (Ziółkowska et al., 2014). Shoots of lentil, soybean, adzuki bean, and alfalfa are shortened on average by $85 \%$ by $20 \mathrm{mM}$ sulfamethazine (Piotrowicz-Cieślak et al., 2010). Drugs contaminating soil inhibit root elongation, on average by $78-84 \%$, but the extent of inhibition depends on the kind of drug, its concentration, and exposure duration (Jin et al., 2009; Migliore et al., 2003; Moullan et al., 2015; Piotrowicz-Cieślak et al., 2010). Even though that the drug concentrations, compared with their lowest tested values, increased 100-fold (Migliore et al., 2003; Moullan et al., 2015), 200-fold (Ziółkowska et al., 2014), and 16 fold in our study, complete inhibition of root growth is not observed. It indicates that even high drug concentrations do not completely inhibit plant growth and development.

The shortening of the roots and shoots is accompanied by a reduction of their fresh mass (Figs 1C, 1D). Leaf biomass of barley and biomass of carrot roots are reduced as a result of exposure of plants to CIP in soil (Eggen et al., 2011). Similarly, oxytetracycline inhibits the biomass of alfalfa shoots and its roots (Kong et al., 2007). Plants growing in soil contaminated with drugs react to the inability of taking up water from soil with tissue dehydration, and consequently a decrease in the dry mass in the analysed under- and above-ground parts of plants. Dry mass of roots and shoots of lupin decreased with increasing concentrations of soluble and insoluble CIP (Figs 1E, $1 F$ ). The same was observed for dry mass of roots and shoots of narrow-leaved lupin growing in soil contaminated with enrofloxacin (Adomas et al., 2013), sulfamethazine (Piotrowicz-Cieślak et al., 2010) and common red soil contaminated with sulfamethazine, oxytetracycline hydrochloride and ciprofloxacin (Liu et al., 2013). It suggests that plants growing in soil contaminated with drugs take up less water. It may be a result of a different water potential between plants and the environment, which makes water uptake more difficult.

Drugs in the soil are absorbed by plants (Santos et al., 2010). The largest amounts of drugs accumulate in roots and leaves and the smallest amounts accumulate in shoots, as they only transport substances to leaves. Since the root is constantly exposed to contaminants, it takes up and accumulates the largest amounts of drugs (Liu et al., 2013). Eight-day-old lupin seedlings (their roots and shoots) took up soluble and insoluble CIP from soil, and CIP content in the plant tissues increased with increasing 
concentrations of the drug in soil. Roots accumulated more soluble and insoluble CIP than shoots. There was 1.5 times more soluble CIP in the analysed organs than its insoluble form (Fig. 2). Similar results were observed in an experiment conducted by Liu et al. (2013). Common reed takes up the largest amounts of soluble salts of drugs (sulfamethazine in the basic form and oxytetracycline and ciprofloxacin as hydrochloride), whereas the accumulation of drugs in plant tissues is positively correlated with their concentration in the substrate. The total content of sulfamethazine, oxytetracycline, and ciprofloxacin in tissues of wetland plant on day 62 of the

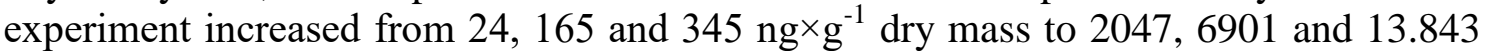
$\mathrm{ng} \times \mathrm{g}^{-1}$ of dry mass, in the samples with the lowest and the highest level of contamination, respectively (Liu et al., 2013). It implies that content of drugs in plant tissues depends on the drug type, its concentration in soil and exposure time. Sulfamethazine content in plant tissues increases 85 times, oxytetracycline -41 times, and ciprofloxacin 40 times. Mol mass of sulfamethazine, oxytetracycline and ciprofloxacin is 300, 460 and 330, respectively, and these values indicate that drug uptake does not depend solely on their mass (Eggen et al., 2011). It is assumed that drug accumulation is the most intensive when they are not too polar or highly hydrophobic. Drug dissolvability and bioaccumulation are defined by index Octanol-Water Partition Coefficient KOW. Log KOW values are generally inversely related to aqueous solubility and directly proportional to molecular weight (U.S. Environmental Protection Agency, 2009). In this study, we used two forms of CIP, soluble and insoluble, that differed in their speed of uptake from soil. We found more of the soluble form in lupin plants but the phytotoxic effect was the same as for the insoluble form. The mol mass for insoluble CIP is 385.82 and for soluble CIP it is 331.34. The $\log$ Kow for CIP is from 0.28 to 2.1 (Halling-Sørensen et al., 2000). Plants take up drugs from soil, using and by water transport and passive absorption Liu et al. (2013) are transported to roots, shoots, and seeds. High content of drugs oil seeds can be a risk for animals and people (Eggen et al., 2011).

Taking up and accumulation of drugs results in alteration of the course of numerous biochemical reactions in plants. It has been found that exposing plants to drugs results in inhibition of chlorophyll biosynthesis (Kong et al., 2007), decreases the concentration of carotenoids in leaves, inhibits photosystem II of photosynthesis (Zhu et al., 2001; Liu et al., 2013) and the production of abscisic acid (Pomati et al., 2004). These are not the only responses of plants to the presence of drugs in the environment. In legume plants treated with sulfamethazine, inhibition of root elongation was accompanied by changes in the activity of cytochrome oxidase in mitochondria and in cytosol. Lupin was particularly sensitive to the drug, which is indicative of its ability to detect soil contamination with sulfamethazine (Piotrowicz-Cieślak et al., 2010).

The activity of peroxidase in roots was lower by $78 \%$ on average than in lupin shoots exposed to soluble CIP (5mM), and by $74 \%$ when plants were exposed to its insoluble form (Figs 3A, 3B). Similarly, increasing concentrations of diclofenac in soil reduce the activity of cytochrome oxidase in root mitochondria of lupin, lentil and pea, with a simultaneous increase in the activity of the enzyme in cytosol (Ziółkowska et al., 2014). A change in the activity of antioxidative enzymes in leaves of sugar cane is also brought about by a mixture of sulfamethazine, oxytetracycline, and ciprofloxacin Liu et al., (2013). The highest concentrations of these drugs inhibit the activity of dismutase and catalase by $53 \%$ and $54 \%$, respectively (Figs 3C, 3D). Although the activity of peroxidase increases at low concentrations, a larger amount of drugs reduces the activity of this 
enzyme. An increase of drug concentrations in soil and drug content in plants leads to a more effective antioxidative protection than in the control sample (Liu et al., 2013).

Deactivation of toxic substances and antioxidative protection (Watanabe et al., 2010) are not the only response of plants to soil contamination. The accumulation in plant tissues of compounds with many hydroxyl groups is also a common defence mechanism (Ortbauer and Popp, 2008). Plants react to changes of humidity and temperature by modification of the concentration of soluble carbohydrates, for example, raffinose family oligosaccharides (RFO) and polyhydroxyalcohols, such as their cyclic forms (cyclitols): myo-inositol, D-chiro-inositol, D-pinitol. Carbohydrates protect cellular structures and proteins with the hydroxyl groups in their structure, which can replace water (Adomas and Piotrowicz-Cieślak, 2004). RFO and galactosyl cyclitols are the basic forms of sugar storage in legume seeds, and their concentration does not usually exceed $20 \%$ of the plant dry weight. RFO is accumulated during natural or forced dehydration of seeds (Piotrowicz-Cieślak et al., 2003). Soluble carbohydrates are helpful in determination of the inhibition of germination and growth of plants (Ziółkowska et al., 2014), because complete decomposition of RFO and galactosyl cyclitols takes place during an early phase of lupin germination (Lahuta et al., 2000). The presence of drugs in soil also affects the content and metabolism soluble carbohydrates in plants. Seedlings of lupin, lentil, and pea exposed to diclofenac at 0.06-12 mM, accumulate D-pinitol, raffinose and stachyose, while containing less glucose, fructose and myo-inositol (Ziółkowska et al., 2014). Commonly occurring in plants, myo-inositol is an important substance in the process of plant growth. It is responsible for signal transduction in plants. Inositol is a precursor of phosphatidylinositol, phytic acid and it is a component of other compounds that take part in alleviating the effects of stress (Loewus and Murthy 2000). Pinitol in fullyhydrated legume plants is the main component of the fraction of soluble carbohydrates (McManus et al., 2000). However, even moderate water stress reduces its concentration, while fructose and sucrose increase their concentration. Sucrose is more effective for membrane protection than reducing sugars (Wang et al., 2013). The current study showed that 8-day old lupin seedlings take up and tolerate higher concentrations of soluble and insoluble CIP than concentrations found in the environment. Inhibition of root and shoot length is probably less harmful to the plants than the increased drug content in the plants that reduces enzyme activity (peroxidise and dismutase), and induces synthesis of D-chiro-inositol, D-pinitol and myo-inositol in seedling roots and shoots (Fig. 4).

\section{Conclusions}

The uptake of two forms of ciprofloxacin (water-soluble and insoluble) by plants was different. There was approximately 1.5 times more ciprofloxacin in seedlings treated with the water-soluble CIP compared to plants affected by the insoluble, free base CIP form. Both CIP forms were phytotoxic to lupin seedlings. Contrary to the preliminary assumptions, insoluble CIP inhibited elongation of roots and shoots nearly as severely as the water-soluble form. The study was carried out on reagent grade chemicals, so it depicts antibiotic-plant interaction in a simplified, reductionist model system. The patterns observed here might be confounded under real world, field conditions by partial transformation of the antibiotics in animal bodies and the presence of a plethora of accompanying molecules in manure. 


\section{REFERENCES}

[1] Adomas, B., Antczak-Marecka, J., Nałęcz-Jawecki, G., Piotrowicz-Cieślak, A. I. (2013): Phytotoxicity of enrofloxacin soil pollutant to narrow-leaved lupin plant. Polish Journal of Environmental Studies 22(1): 71-76.

[2] Adomas, B., Piotrowicz-Cieślak, A. I. (2004): Amino acid composition, hemicellulose and soluble sugars content in narrow- leaves lupin seeds (Lupinus angustifolius L.) under the effect of Reglone Turbo 200 SL. - Electronic Journal of Polish Agricultural Universities, Agronomy 7(2): 1-9.

[3] Bakalovic, N., Passardi, F., Ioannidis, V., Cosio, C., Penel, C., Falquet, L., Dunand, C. (2006): PeroxiBase: A class III plant peroxidase database. - Phytochemistry 67(6): 534539.

[4] Caco, A. I., Varanda, F., Pratas de Melo, M .J., Dias, A. M. A., Dohrn, R., Marrucho, I.M. (2008): Solubility of antibiotics in different solvents. Part II. Non-hydrochloride forms of tetracycline and ciprofloxacin. - Industrial \& Engineering Chemistry Research 47(21): 8083-8089.

[5] Cesarino, I., Araújo, P., Paes Leme, A. F., Creste, S., Mazzafera, P. (2013): Suspension cell culture as a tool for the characterization of class III peroxidases in sugarcane. Plant Physiology and Biochemistry 62: 1-10.

[6] Cheng, G. Y., Liu, J., Xuan, T. M., Mei, L. C., Wu, G. R. (2012): Activity, thermostability and isozymes of superoxide dismutase in 17 edible mushrooms. Journal of Food Composition and Analysis 26(1-2): 136-143.

[7] Ding, C., He, J. (2010): Effect of antibiotics in the environment on microbial populations. Applied Microbiology and Biotechnology 87(3): 925-941.

[8] Drugbank DB00537 - http://www.drugbank.ca/drugs/DB00537\#references

[9] Eggen, T., Asp, T. N., Grave, K., Hormazabal, V. (2011): Uptake and translocation of metformin, ciprofloxacin and narasin in forage- and crop plants. - Chemosphere 85(1): 26-33.

[10] Golet, E. M., Strehler, A., Alder, A. C., Giger, W. (2002): Determination of fluoroquinolone antibacterial agents in sewage sludge and sludge-treated soil using accelerated solvent extraction followed by solid-phase extraction. - Analytical Chemistry (74): 5455-5462.

[11] Halling-Sørensen, B., Holten Lützhøft, H. C., Andersen, H. R., Ingerslev F. (2000): Environmental risk assessment of antibiotics: comparison of mecillinam, trimethoprim and ciprofloxacin. - Journal of Antimicrobial Chemotherapy 46, Supplement S1, 53-58.

[12] ISTA (1999): International rules for seed testing. - Seed Science and Technology Supplement 27(1).

[13] Jin, C., Chen, Q., Sun, R., Zhou, Q., Liu, J. (2009): Eco-toxic effects of sulfadiazine sodium, sulfamonomethoxine sodium and enrofloxacin on wheat, Chinese cabbage and tomato. - Ecotoxicology 18(7): 878-885.

[14] Kemper, N. (2008): Veterinary antibiotics in the aquatic and terrestrial environment. Ecological Indicators (8):1-13.

[15] Kong, W .D., Zhu, Y. G., Ling, Y. C., Zhang, J., Smith, F. A., Yang, M. (2007): Uptake of oxytetracycline and its phytotoxicity to alfalfa (Medicago sativa L.). - Environmental Pollution 147 (1): 187-193.

[16] Lahuta, L., Górecki, R., Michalczyk, D., Piotrowicz-Cieślak, A. (2000): Alpha-Dgalactosidase activity in stored yellow lupin (Lupinus luteus L.) seeds. - Electronic Journal of Polish Agricultural Universities, Agronomy 3(1): 1-9.

[17] Lillenberg, M., Litvin, S. V., Nei, L., Roasto, M., Sepp, K. (2010): Enrofloxacin and ciprofloxacin uptake by plants from soil. - Agronomy Research 8(1): 807-814.

[18] Liu, L., Liu, Y., Liu, C., Wang, Z., Dong, J., Zhu, G., Huang, X. (2013): Potential effect and accumulation of veterinary antibiotics in Phragmites australis under hydroponic conditions. - Ecological Engineering 53: 138-143. 
[19] Loewus, F. A., Murthy, P. P. N. (2000): Myo-Inositol metabolism in plants. - Plant Science 150(1): 1-19.

[20] Lowry, O. H., Rosebrough, N. J., Farr, A. L., Randall, R. J. (1951): Protein measurement with folin phenol reagent. - Journal of Biological Chemistry 193(1): 265275.

[21] Martínez-Carballo, E., González-Barreiro, C., Scharf, S., Gans, O. (2007): Environmental monitoring study of selected veterinary antibiotics in animal manure and soils in Austria. - Environmental Pollution 148: 570-579.

[22] McManus, M. T., Bieleski, R. L., Caradus, J. R., Barker, D. J. (2000): Pinitol accumulation in mature leaves of white clover in response to a water deficit. Environmental and Experimental Botany 43(4): 11-18.

[23] Migliore, L., Cozzolino, S., Fiori, M. (2003): Phytotoxicity to and uptake of enrofloxacin in crop plants. - Chemosphere 52(7): 1233-1244.

[24] Moullan, N., Mouchiroud, L., Houtkooper, R. H., Auwerx J. (2015): Tetracyclines disturb mitochondrial function across eukaryotic models: A call for caution in biomedical research. - Cell Reports 10: 1681-1691.

[25] Noiraud, N., Maurousset, L., Lemoine, R. (2001): Transport of polyols in higher plants. - Plant Physiology and Biochemistry 39(9): 717-728.

[26] Oliphant, C. M. Green, G. M. (2002): Quinolones: a comprehensive review. - American Family Physician 65: 455-464

[27] Ortbauer, M., Popp, M. (2008): Functional role of polyhydroxy compounds on protein structure and thermal stability studied by circular dichroism spectroscopy. - Plant Physiology and Biochemistry 46(4): 428-434.

[28] Pailler, J., Krein, A., Pfister, L., Hoffmann, L., Guignard, C. (2009): Solid phase extraction coupled to liquid chromatography-tandem mass spectrometry analysis of sulfonamides, tetracyclines, analgesics and hormones in surface water and wastewater in Luxembourg. - Science of the Total Environment 407(16): 4736-4743.

[29] Passardi, F., Longet, D., Penel, C., Dunand, C. (2004): The class III peroxidase multigenic family in rice and its evolution in land plants. - Phytochemistry 65(13): 1879-1893.

[30] Piotrowicz-Cieślak, A. I. (2005): Changes in soluble carbohydrates in yellow lupin seed under prolonged storage. - Seed Science and Technology 33(1): 141-145.

[31] Piotrowicz-Cieślak, A. I., Adomas, B. (2012): Herbicide phytotoxicity and resistance to herbicides in legume plants. - In: R. Alvarez-Fernandez (Ed.) Herbicides Environmental Impact Studies and Management Approaches, InTech, 19-44.

[32] Piotrowicz-Cieślak, A. I., Adomas, B., Nałecz-Jawecki, G., Michalczyk, D. J. (2010): Phytotoxicity of sulfamethazine soil pollutant to six legume plant species. - Journal of Toxicology and Environmental Health, Part A 73 (17-18): 1220-1229.

[33] Piotrowicz-Cieślak, A. I., Lopez, P. M. G., Gulewicz, K. (2003): Cyclitols, galactosyl cyclitols and raffinose family oligosaccharides in Mexican wild lupin seeds. - Acta Societatis Botanicorum Poloniae 72(2): 109-114.

[34] Pomati, F., Netting, A. G., Calamari, D., Neilan, B. A. (2004): Effects of erythromycin, tetracycline and ibuprofen on the growth of Synechocystis sp. and Lemna minor.Aquatic Toxicology 67(4): 387-396.

[35] Rontein, D., Basset, G., Hanson, A D. (2002): Metabolic engineering of osmoprotectant accumulation in plants. - Metabolic Engineering 4(1): 49-56.

[36] Rydzyński, D., Piotrowicz-Cieślak, A. I., Grajek, H., Michalczyk, D. J. (2017): Instability of chlorophyll in yellow lupin seedlings grown in soil contaminated with ciprofloxacin and tetracycline. - Chemosphere 184: 62-73.

[37] Santos, L. H., Araújo, A. N., Fachini, A., Pena, A., Delerue-Matos, C., Montenegro, M.C. (2010): Ecotoxicological aspects related to the presence of pharmaceuticals in the aquatic environment. - Journal of Hazardous Materials 175(1-3): 45-95. 
[38] Sikorski Ł., Adomas B., Dobiesz M., Baciak M., Piotrowicz-Cieślak A. I. (2014): Morphological and biochemical responses of Lemna minor L. (common duckweed) to ciprofloxacin. - Fresenius Environmental Bulletin 23(2): 363-371.

[39] U.S. Environmental Protection Agency

(2009): http://www.epa.gov/osw/inforesources/data/br09/br2009rpt.pdf

[40] Wang, L., Cui, N., Zhang, K. Y., Fan, H. Y., Li, T. L. (2013): Research advance of sucrose phosphate synthase (SPS) in higher plant. - International Journal of Agriculture and Biology 15(6): 1221-1226.

[41] Watanabe, L., De Moura, P. R., Bleicher, L., Nascimento, A. S., Zamorano, L. S., Calvete, J. J., Sanz, L., Pérez, A., Bursakov, S., Roig, M. G., Shnyrov V. L., Polikarpov I. (2010): Crystal structure and statistical coupling analysis of highly glycosylated peroxidase from royal palm tree (Roystonea regia). - Journal of Structural Biology 169(2): 226-242.

[42] Zhu. H., Chen, X., Pan, X., Zhang, D. (2011): Effects of chloramphenicol on pigmentation, proline accumulation and chlorophyll fluorescence of maize (Zea mays) seedlings. - International Journal of Agriculture and Biology 13(5): 677-682.

[43] Ziółkowska, A., Piotrowicz-Cieślak, A. I., Rydzyński, D., Adomas, B., Nałęcz-Jawecki, G. (2014): Biomarkers of leguminous plant viability in response to soil contamination with diclofanac. - Polish Journal of Environmental Studies 23(1): 263-269. 